\title{
AN INFRARED CAMERA BASED ON A LARGE PtSi ARRAY
}

\author{
I. S. Glass, K. Sekiguchi and \\ South African Astronomical Observatory \\ Y. Nakada \\ Kiso Observatory, Inst. of Astronomy, Univ. of Tokyo
}

\begin{abstract}
A large-format PtSi array (effectively $1 \mathrm{k} \times 0.5 \mathrm{k}$ pixels) has been incorporated into an infrared camera intended for survey work using the $0.75-\mathrm{m}$ telescope at Sutherland. The array is very uniform and almost free of cosmetic defects. The camera, its electronics and the operational procedures that we use are described, together with the methods of data reduction. The limiting magnitudes that can be observed in the JHK broad bands are given.
\end{abstract}

\section{INTRODUCTION}

This instrument is a joint project between the South African Astronomical Observatory, the Institute of Astronomy of the University of Tokyo and the National Astronomical Observatory of Japan. It has provision for up to six filters, normally including the J(1.25), $\mathrm{H}(1.65), \mathrm{K}(2.2)$ and $\mathrm{K}^{\prime}(2.15)$ bands. The $\mathrm{K}^{\prime}$ filter is one which omits the long-wave part of the regular $\mathrm{K}$ band, i.e. that which contributes most to the thermal background arising from the telescope and sky.

Because of its large format but low quantum efficiency, the camera is expected to be employed mainly for survey projects. For example, it is being used to survey a number of fields close to the galactic center $\left(A_{v} \sim 30\right.$ mag) to verify the apparent build-up of luminous stars towards the center noted by Catchpole, Whitelock and Glass (1990). This requires exposures in two or three colors to estimate the interstellar reddening along the line of sight. While at $\mathrm{K}$ the extinction will be about 2.5 mags, at $\mathrm{J}$ it is about 7.5 mags and only foreground objects are visible.

A further project concerning the galactic center is to monitor fields at a single wavelength reasonably frequently to pick out variable stars such as miras which are expected to be particularly numerous. The $\mathrm{K}$ band is again expected to be the most useful for this purpose in spite of the high foreground extinction.

The same techniques will be applied to survey certain fields in the Magellanic Clouds for luminous AGB stars and variables, though these appear to be close to the limit achievable with the 0.75-m telescope. 


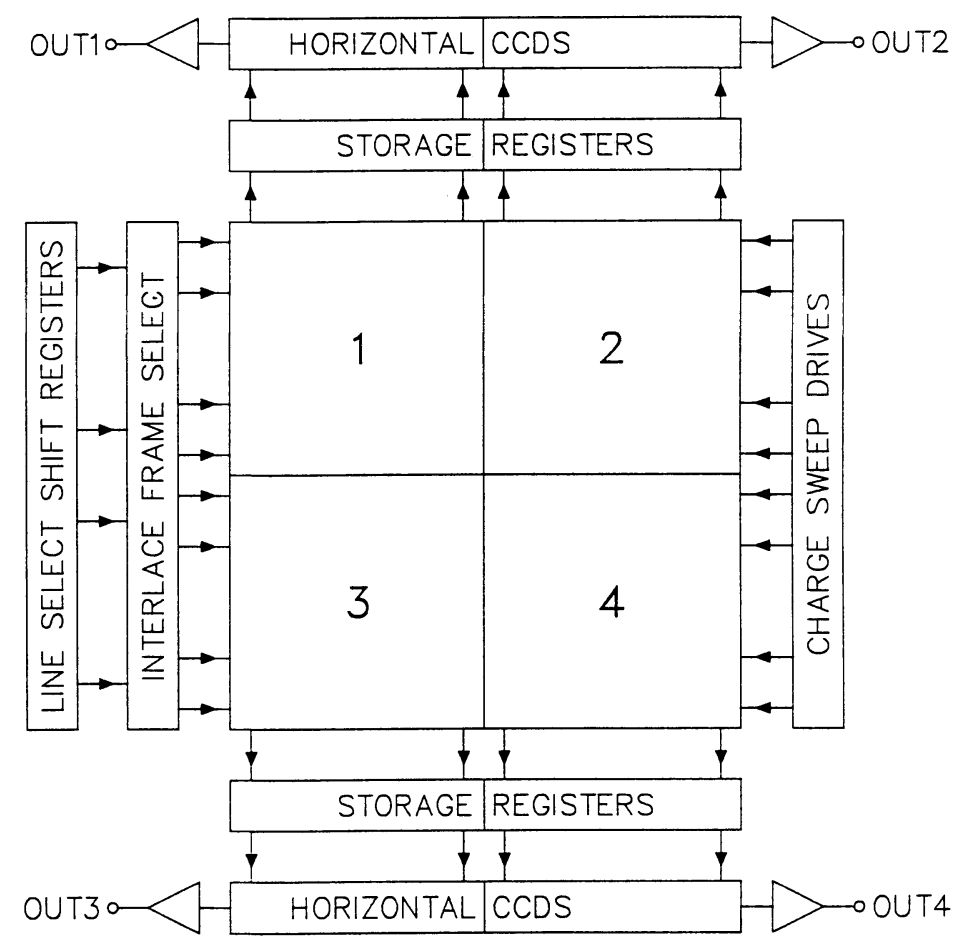

Fig. 1. Functional diagram of the chip. It has four independent sectors.

The camera is also being used to examine the late-type stellar content of globular clusters. Here again, the large format of the detector outweighs its low quantum efficiency.

In addition, the Comet Shoemaker-Levy impact with Jupiter during July 1994 turned out to be an ideal subject for the PtSi camera. Some of the observations were made in late afternoon more than an hour before sunset.

\section{CHARACTERISTICS OF THE CHIP}

The chip is from the family described by Kimata et al. (1992). It is of monolithic construction, using proven silicon techniques, so that it has few defective pixels. The detectors are PtSi Schottky-barrier type. The device has four independent sectors (see Fig. 1), each of which may be regarded as a separate 520 × 260-pixel detector. There are no dead spaces between the sectors. The readout commences simultaneously in the four outer corners of the chip and progresses line by line until the center is reached. In each quarter, the charges from pairs of lines are switched one pair at a time into four-phase vertical CCDs which "sweep" them into storage registers. Because only one pair is being swept down at a time, the operation can be 
carried out many times to overcome any charge transfer inefficiency effects due to the narrowness of the channels. Following the charge sweep operation, the storage registers are emptied into conventional four-phase horizontal CCDs for readout via floating-gate amplifiers.

The pixel spacing is 17 microns in each direction, with $53 \%$ fill factor. Because the lines are read out in pairs, the pixel size is effectively 17 microns in the horizontal direction by 34 in the vertical. The quantum efficiency of the device varies from about $6 \%$ at $\mathrm{J}$ to about $2 \%$ at $\mathrm{K}$. About $1 \%$ drop in response (non-linearity) is noticed when the wells contain about 600,000 electrons.

The problem mentioned by Glass et al. (1994), in an earlier report on this device, namely that the background varied slightly from line to line, has been eliminated by a change in the waveforms applied. In effect, the vertical CCDs are now operated separately from the horizontal ones (they were previously interleaved). The unevenness arose due to very slight irregularities in the waveform timing, induced by the assynchronous nature of the Transputer link which transmits the data after each A/D conversion.

\section{CONTROLLER}

The chip is driven by one of the SAAO/RAL Transputer-based controllers described by Glass et al. (1994). This consists of a number of circuit cards in a Eurocrate. The clock waveforms are controlled directly from software and their timing is governed by the Transputer which can output words by DMA (direct memory access) every $200 \mathrm{~ns}$. D/A converters on two "clock" cards generate the actual pulses to be applied as well as a number of DC supplies. The outputs from the chip first go to differential preamplifiers mounted on the dewar and then to four "video cards" which contain the 16-bit A/D converters. The digitized data are sent in Transputer link format (eight bytes or four 16-bit words at a time) over optical fibers to the data acquisition computer from which the observer works and where the data are stored. Other modules in the controller are used to operate the filter wheel and to monitor the temperature of the chip. The Transputer is programmed in OCCAM and assembler and the program is stored on an EPROM.

\section{DATA ACQUISITION COMPUTER}

The observer's computer acts as a terminal to the controller. Simple one-character messages sent to the controller are used to initiate readouts of various kinds, change filters, measure temperatures etc. The composition of an exposure sequence in terms of pre-wipes and actual readout is controlled from the data acquisition computer. The exposure sequence proper is a pre-wipe followed by an exposure time measured in the Transputer and, finally, the readout with A/D conversion. The data are sorted into their correct order in a 1040 x 520 matrix as they arrive. After the readout they are recorded with their FITS header onto disk. They are then scaled and displayed according to their maximum and minimum values and a histogram of pixel values is also placed on the screen.

The processor is a $486 / 50 \mathrm{MHz}$ PC; there are $8 \mathrm{MB}$ of memory and $340 \mathrm{MB}$ of disk space. Pictures are displayed on a super-VGA monitor in 1028 x 768 256-color mode. An auxiliary black and white monitor is used for alphanumeric data. A set of programs similar to PC-VISTA 
(Treffers and Richmond 1989) enable some on-line reduction to be performed. In particular, files can be displayed and images analyzed for focussing and crude photometry. The data are saved on a DAT tape at the end of the night. The PC uses Gnu C for 32-bit addressing.

A special data acquisition program was written for the Comet Shoemaker-Levy/Jupiter event to allow continuous exposures with readouts as frequently as every ten seconds. Because there were of necessity no pre-exposure wipes, a (mainly) bias background frame had to be subtracted from each exposure to reveal the planet. Only one quarter of each frame was recorded on disk so that up to three hrs coverage could be obtained without interruption.

\section{OPTICAL MATCHING}

The camera is used on the 0.75-m telescope at Sutherland at an effective focal ratio of $\mathrm{f} / 16$ and a scale of 17 arcsec per $\mathrm{mm}$. The diffraction limit of this telescope at 2.2 microns is 0.7. There is some lateral spherical aberration due to the displacement of the Cassegrain focus below



Fig. 2. Cross-section of lower part of cryostat. The filter wheel has space for six filters of 30 $\mathrm{mm}$ diameter, but one position is normally blanked off for use as a shutter. 
its design position. The best achieved FWHM of the image is 0.8 to 0.9 in very good seeing. The scale of the image amounts to 3.3 pixels per arcsec. Such oversampling was felt to be necessary because of the $53 \%$ fill factor of the chip.

There is no field imaging, and hence no position in which to place a cold "Lyot" stop. This was at least in part due to a lack of design information for a lens having the necessary resolution and wavelength range. With the present set of cold baffles, the detector "sees" around 16 times more solid angle of telescope and sky than desirable. This is not a very serious problem at $\mathrm{J}$ and $\mathrm{H}$, but it restricts $\mathrm{K}$ exposures to little more than five minutes during the warm part of the year. A longer cold baffle, giving a reduction of two to three times in the background, is to be installed soon. The $\mathrm{K}$ signal-to-noise ratio should then improve by a few tenths of a magnitude.

\section{TEMPERATURE}

The detector needs to be maintained at $60^{\circ} \pm 2 \mathrm{~K}$, representing a compromise between excessive dark current and charge transfer inefficiency. Using a simple micrometer valve on a large vacuum pump, the temperature stays within $0.5^{\circ} \mathrm{K}$ during the night.

\section{SIGNAL ANALYSIS}

There is no mechanical shutter since it would have to be cold as well as fast in order to be useful. Instead, a "dummy" readout (taking $1.6 \mathrm{~s}$ ) defines the start of an exposure. The real readout $(6.25 \mathrm{~s})$ defines the end of the exposure. This approach requires that the data from each frame must be corrected to allow for the change of exposure time with position.

The signal from the detector results from (a) photons truly from the field (b) photons from sky and telescope surrounding telescope exit pupil (c) dark current $\left(\sim\right.$ seven electrons $/ \mathrm{sec}$ at $60^{\circ}$ K) (d) Spurious radiation from the amplifiers (e) "Trapped" electrons depending on the recent "history" of the chip.

The amplifier radiation is the most serious of the spurious components. The amplifiers are situated in the corners of the chip and, if driven in the manner recommended for normal high-speed readout, they would saturate the scene immediately. A number of methods are used to reduce this problem.

a) The output amplifiers are turned on only when the readout commences.

b) The readout time is minimized - at $6.25 \mathrm{~s}$ its lower limit is mainly determined by the speed of the Transputer link and its protocol.

c) The load resistors of the output emitter followers, across which the signals are measured, are kept as high as practicable to reduce the current in the device.

d) The bias of the first stage of the output amplifiers (each amplifier is a three-transistor device) is kept as low as possible consistent with reliable operation.

With these precautions, the effective read noise towards the center of the chip is equivalent to about 30 electrons. The first pixels to be read out are those nearest the output amplifiers and the spurious charge does not have enough time to build up. The worst remaining effects of 
amplifier emission are visible on the outer ends of the last lines to be read and affect the baseline noise level, causing difficulties with the DoPHOT (Schecter, Mateo and Saha 1993) reduction procedure which normally assumes a uniform noise level.

The chip is given ten dummy readouts before an exposure to clear it of charge from the previous exposure. However, it is found that small numbers of pixels in the extreme corners are not cleared consistently. It is believed that this problem is caused by the saturation of parts of the chip close to the output amplifiers during the preceding readouts. (Note added later: $\mathrm{Dr}$ $M$. Ueno has made some suggestions about changes to the "dummy" readout waveforms which should help to solve this problem.)

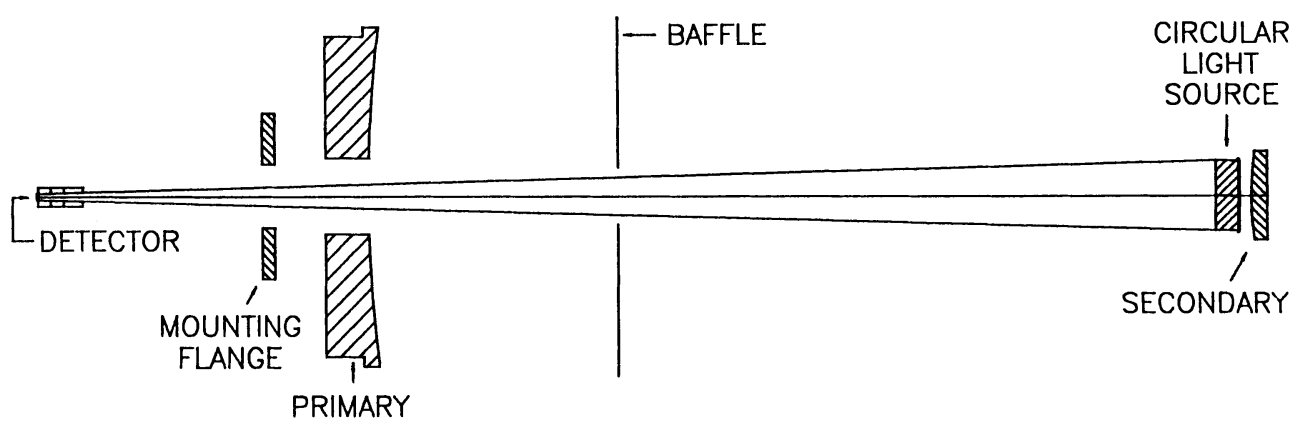

Fig. 3. Flatfield arrangement on the $0.75-\mathrm{m}$ telescope.

\section{FLATFIELDS}

The over-wide pupil denies the use of the sky or screens within the dome for flatfielding purposes. The solution that we have adopted is to place a flat circular diffuse light source in front of the secondary (close to the position of, and having the same apparent size as the exit pupil). A baffle along the tube cuts of light from outside the exit pupil. The response of the detector is measured with the light on and off to determine the difference which is then taken as representative of light from the telescope pupil.

\section{REDUCTION PROCEDURE}

The basic reduction procedure is to: (a) Correct the fields for uneven exposure time, (b) Divide by the appropriate flatfield, (c) Subtract the appropriate sky frame, (d) Measure the images, for example by DoPHOT (Schecter et al. 1993) and (e) Standardize .

\subsection{Median Averaging of Frames}

Because of the high background at $\mathrm{K}$ and to some extent at the other wavelengths, it is generally necessary to subtract sky frames taken under the same conditions as the object 
exposures. This not only removes the extraneous background, but also that due to the dark current and the output amplifiers. Unfortunately, the survey fields are large and often crowded, so that it is usually impossible to find one devoid of stellar objects nearby. In addition, the time taken for a survey is doubled. However, it is possible to construct sky frames from a number of survey frames by median averaging (pixel-by-pixel). Usually, at least five frames are necessary to avoid chance coincidences of stellar positions.

The formation of median averaged frames is not completely straightforward. The sky background in the near-infrared is primarily due to $\mathrm{OH}^{-}$emissions originating high in the atmosphere and is variable by several percent on time scales of a few minutes (Ramsay, Mountain and Geballe 1992). At wavelengths longer than about 2.3 microns, the thermal background from the atmosphere and telescope become dominant. These also show slow fluctuations on time scales comparable to those used for exposures.

To remove the effect of the fluctuations we treat them at present as if they are completely uniform over the entire frame. A group of exposures is first averaged pixel-by-pixel in the ordinary way. The average frame is subtracted in turn from each individual on a histogram is made of each difference frame and the commonest pixel value is used to calculate an offset to correct the original frames to a standard background level. The median average is then obtained and used to subtract the background from each corrected frame, which is then cleaned to remove bad pixels etc. and passed to DoPHOT for further analysis.

It is intended to use a procedure similar to that described by Balona (1994) for obtaining accurate photometry and surveying for variable stars.

\subsection{Accuracy Achieved}

Calculations of the number of photons that should reach the detector from a star of a given magnitude agree reasonably well with the actual numbers measured. From the observed backgrounds due to thermal radiation and the read-out amplifiers it is immediately obvious that they control the limiting magnitudes that can be achieved.

So far, very limited quantities of data have been analyzed in detail. The same quarter of two five-minute galactic center $\mathrm{K}$ pictures taken at different times with fairly good seeing conditions ( $\sim 1.5 \mathrm{~s}$ FWHM) have been processed using DoPHOT and compared. It should be noted, however, that our implementation of DoPHOT has not yet been fully optimized for this task. Four stars brighter than $\mathrm{K}=9$ have photometry differing by $<=1 \%$. By $\mathrm{K} \sim 11$, the magnitude differences are around 10\%; the exact figure is uncertain as the data are somewhat contaminated by misidentifications of objects between the two frames. Theoretically, under similar seeing conditions, an error of about 0.03 mag would be expected at $\mathrm{K}=11$. The larger apparent error may be due to the fact that the flatfields were each derived from single exposures and the median sky frames from only five exposures each, including one near the galactic center which can be expected to be excessively crowded.

The limiting magnitudes in the $\mathrm{J}$ and $\mathrm{H}$ bands for similar errors are controlled by the noise due to amplifier radiation. Calculations indicate that at $\mathrm{J}$ we should reach 14.7 and at $\mathrm{H} 13.5$ at $\pm 10 \%$ error. 


\section{FUTURE WORK}

The limiting magnitude at $\mathrm{K}$ will be made a few tenths of a magnitude fainter by the addition of a longer cold baffle to reduce the extraneous radiation from the telescope and it is expected that this will be installed during September 1994. The possibility of introducing relay optics and a cold pupil stop are also being investigated. General improvements will be obtained by taking more flatfield frames during each observing run. It appears that the flatfield characteristics of the array are quite stable.

Additional improvement may be possible in the suppression of amplifier radiation and experiments are planned in this area. The effects of changes in the clocking waveforms remain to be investigated.

\section{ACKNOWLEDGMENTS}

We wish to thank Mr D. B. Carter for his work on the electronics of the camera; Dr L. A. Balona and Dr J. W. Menzies for their patient help with programming queries; Dr M. Ueno, University of Tokyo, for information concerning the properties of the chip; Mr W. Pearson, $\mathrm{Mr} \mathrm{D}$. Weir and other members of the SAAO technical staff for their help at various stages of this project.

\section{REFERENCES}

Balona, L. A. 1995 in IAU Symposium No. 167,New Developments in Array Technology and Applications, A. G. D. Philip, K. A. Janes and A. R. Upgren, eds., Kluwer Academic Pub. Dordrecht, p. 187

Catchpole R. M., Whitelock P. A. and Glass I. S. 1990 MNRAS 247, 479

Glass, I. S., Carter, D. B., Sekiguchi, K. and Nakada, Y. 1994 in Infrared Astronomy with Arrays: The Next Generation, I. S. McLean, eds., Kluwer Academic Pub., Dordrecht, p. 285

Kimata, M., Yutani, N., Tsubouchi, N. and Seto, T. 1992 SPIE 1762, Infrared Technology XVIII

Ramsay S. K., Mountain C. M. and Geballe T. R. 1992 MNRAS 259, 751

Schecter, P. L., Mateo, M. and Saha, A. 1993 PASP 105, 1342

Treffers, R. R. and Richmond, M. W. 1989 PASP 101, 725

\section{DISCUSSION}

FAZIO: Are the $1040 \times 1040 \mathrm{PtSi}$ arrays commercially available?

GLASS: I believe they are developmental types released only to the Japanese astronomers.

D'ODORICO: In view of the dramatic development in the size and capabilities of InSb and $\mathrm{HgCdTe}$ array, do you think that $\mathrm{PtSi}$ arrays will still be competitive in the future?

GLASS: If the QE of PtSi chips can be improved dramatically I think they will remain competitive, especially because of their high cosmetic quality and relative uniformity. 